\title{
Comunicación, Gerencia y Futuro: Una interpretación posmoderna
}

\author{
Rojas Vera, Luis Rodolfo \\ Arapé Copello, Elizabeth \\ Mujica Miguel \\ Rodríguez, Ramón \\ L ara Cortés, Carmen Ofelia \\ Durand, Efraín \\ Ugas, Gabriel
}

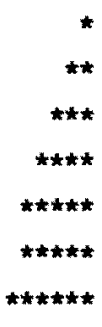

\section{Resumen}

El artículo trata el tema de la tríada Comunicación, Gerencia y Futuro en las organizaciones como un campo de interés para investigadores de cualquiera de estas áreas. $E \mid$ estudio se adelanta desde una perspectiva posmoderna esto representa un reto a la interpretación interdisciplinaria. Centra la atención en cinco problemas fundamentales como rutas para interpretar las interrelaciones entre los elementos de la tríada. Los cinco problemas principales son: el cambio incremental; el gobierno de la infocomunicación; la crisis de los paradigmas; el juego de las paradojas y el déficit conceptual.

Palabras clave: Comunicación, gerencia, futuro, cambio, información, crisis, paradojas, oportunidades y retos.

Recibido: 99-02-23. Aceptado: 99-06-23

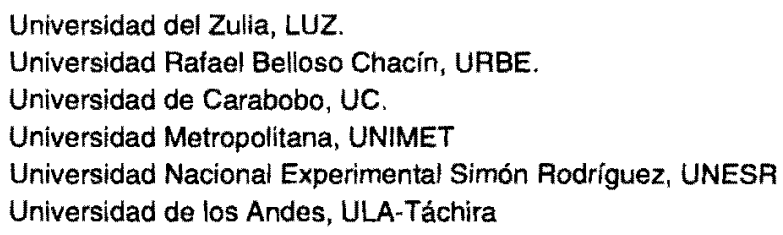




\section{Communication, Management and the Future: A Post-Modern Interpretation}

\section{Abstract}

This article deals with the triad of Communications, Management and Future in management, as a field of interest for research in any or all of these areas. The study develops from a post-modern conception in that it represents a challenge to interdisciplinary interpretation. Attention is centered on five fundamental problems that become routes by which to interpret the interrelations between the elements of the triad. The five fundamental problem areas are: incremental change; the control of info-communications; paradigmatic crisis; the interplay of the various paradoxes; and conceptual deficit.

Key words: Communications, management, future, change, information, crisis, paradoxes, opportunities and challenges.

\section{Introducción}

En este artículo nos proponemos discutir algunas cuestiones sobre la tríada "Comunicación, Gerencia y Futuro" en el ámbito de las organizaciones intentando una interpretación posmoderna'. Las grandes interrogantes que orientan el problema planteado en este trabajo pueden sintetizarse en las siguientes ideas: ¿Cuál puede ser la agenda para tratar los nuevos cambios en el mundo organizacional bajo el impacto de las comunicaciones? ¿Qué interpretación posmoderna podemos efectuar sobre los cambios organizacionales? ¿Cómo se interrela- ciona la comunicación y la gerencia hacia el futuro de las organizaciones?

Los autores del articulo han constituido un equipo de investigación en red relacionado con ambos proyectos, y están conscientes del riesgo que representa efectuar estudios desde perspectivas que no parecen muy precisas, desde fronteras que no son tan claras, y con ideas que no suelen ser habituales, al menos en la cultura gerencial tradicional.

\section{Metodología}

El trabajo se desarrolló mediante la integración de tres tipos de esfuerzos: 1)

1 El trabajo constituye un avance de investigación que da cuenta de dos proyectos vinculados1) "Comunicación, Gerencia y Futuro" (CONDES 02469.97), Y 2) "Impacto de las Corrientes Posmodernas en la Cultura Organizacional (IMPOSMO) (CONICIT-G9700071). 
revisión teórica, 2) panel de entrevistas, y 3) discusión en Seminarios de postgrado. La revisión teórica trató básicamente la literatura relacionada en las referencias y se orientó sobre tres preocupaciones básicas: 1) la integración de una visión prospectiva de las relaciones entre comunicación y gerencia en las organizaciones, que comprende una visión amplia, $y$, si se desea, compleja y transdiciplinaria; 2) los cambios en el entorno y los cambios en las organizaciones bajo la óptica de la sociedad de las comunicaciones; y 3) las nuevas agendas para el tema de las comunicaciones en torno al mundo de las organizaciones, es decir, las organizaciones obsenadas bajo el paradigma de las sociedad de las comunicaciones.

En el panel de entrevistas participaron algunos Profesores e Investigadores de Programas de Maestría y Doctorados desarrollados en Venezuela que estudian problemas relacionados con la Comunicación y la Gerencia. La selección fue intencional, no más de un representante por programa en atención a su preocupación y/o contribución al estudio de las relaciones comunicación-gerencia abarcando un total de siete entrevistas que se realizaron entre finales de 1997 y finales de 1998. Las entrevistas se registraron en cintas sonoras y algunas en videos. Se utilizó la técnica de la entrevista en profundidad focalizada siguiendo las recomendaciones de autores como Schatzman y Strauss (1973), Merton et al., (1990), Millar et al., (1992), y, entre otros, Valles (1997). En general, se dejó al interlocutor la libertad para el desarrollo de las respuestas. Los datos se interpretaron en un sentido cualitativo atendiendo fundamentalmente a la frecuencia de contenidos y al destacado de ideas centrales. Tres preguntas fundamentales guiaron el diálogo con los expertos: 1) ¿Cómo interrelacionan la comunicación y la gerencia en las actuales organizaciones?, 2) ¿Qué importancia puede tener para la gerencia en las organizaciones del futuro la cuestión de la comunicación?, y, 3) ¿Cuáles son los nuevos horizontes que se avecinan en la relación comunicación y gerencia?

Estos dos insumos, en parte, se discutieron y retroalimentaron en varios cursos de postgrado, y especialmente en los siguientes tres Seminarios: Seminario Doctoral "Comunicación, Gerencia y Futuro" en el doctorado en Ciencias Sociales de LUZ-UCV (1997-1998), Seminario Doctoral "Información y Estrategia Empresarial" en la Universidad Simón Rodriguez (1998-1999), y el Seminario de "Comunicación Organizacional" en la Maestría en Ciencias de la Comunicación, (LUZ-1999). Por medio de las discusiones imentamos interpretar los problemas de la tríada "Comunicación, Gerencia y Futuro", sin un guión preestablecido. E resultado de estas reflexiones que hoy presentamos lo consideramos como un conjunto de signos que sintetizamos en cinco grandes problemas como rutas para la interpretación de las oportunidades y retos que nos plantea la tríada " $\mathrm{Co}$ municación, Gerencia y Futuro" en la organización,

Aunque la idea es continuar una segunda ronda de entrevista, en una especie de método Delphi, por ahora surgen conclusiones preliminares que representan dos extremos de opiniones o tendencias: 1) el que indica que hoy los cambios se generan principalmente desde la 
Rojas, L.; Arapé, E.; Mujica, M.; Rodríguez, R.; Lara, C.; Durand, E.; Ugas, G.

gerencia, fundamentalmente administrativa (en la que la comunicación es un instrumento) y desde la que se direcciona el futuro, y, 2) el que indica que hacia el futu-

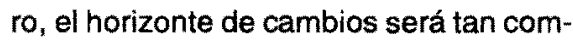
plicado que la gerencia actual podría ser deficitaria, al tiempo que la comunicación, principalmente tecnológica, incrementaria sus capacidades transformacionales. Pudiéramos decir que la primera tendencia es la más cercana a la corriente clásica o tradicional, y que se centra en la racionalidad administrativa de realidades previsibles y manejables. La segunda tendencia trata de desligarse de esquemas tradicionales y destaca las transformaciones, desde la información y la comunicación como epicentro de la sociedad actual. He aqui pues dos lógicas: la lógica administrativa y la lógica comunicacional como paradigmas predominantes ( $y$ no excluyentes) en la concepción y dinámica de la organización.

\section{La perspectiva posmoderna}

Al pensar en las organizaciones, muchos procesos relacionados con la gerencia y la comunicación parecen claros y estables, modelables y previsibles. La razón es que ha imperado un enfoque epistemológico positivista para interpretar a la organización, su gerencia, su comunicación, y hasta su futuro: experimentación, prueba de hipótesis, cuantificaciones, demostraciones de plena certidumbre, linealidad, direccionalidad, y previsibilidad. Precisamente porque tanto la empresa moderna, como su gerencia y su comunicación, han crecido bajo la ciencia moderna. Así, tenemos, por un lado, que la gerencia se ha desarrollado como tal en este siglo y que sus principales paradigmas están impregnados de la ciencia moderna, positiva, de alta racionalidad, constituyendo una metáfora de la mecánica en la que poco se admite la complejidad, las paradojas, las incertidumbres o la transversalidad. La comunicación, por otro lado, suele ser percibida, dentro de las organizaciones, como un instrumento de la gerencia, pesando tanto las visiones instrumentales y funcionales que resulta dificil una comprensión del sentido integral de la comunicación al interior de las organizaciones.

Pero el mundo organizacional se ha vuelto complejo, y ya no es lo que había sido, al menos en su percepción e interpretación. Hemos ingresado a una era más allá de aquella modernidad, a una era que diversos autores han explicado como postmodernidad o posmodernidad (Baudrillard, 1968; Lyotard,1979; Foucault, 1994; Foltari y Lanz, 1998; Derrida, 1972; Habermas, 1981; y, entre otros, De Sousa, 1996) precisamente porque, entre diversas razones, se ha comenzado a superar el esquema mecánico de la interpretación, aceptando el advenimiento de crisis de paradigmas y de desplazamientos de limites tradicionales. Se reconoce un quiebre histórico y se comienza a admitir la importancia de la mutidimensionalidad, de la complejidad (Morín, 1990) y la transversalidad que afectan desde lo social hasta las organizaciones empresariales. La vida, el saber, las organizaciones y su conducción se han vuelto, cada día más complejas, inciertas, paradójicas, e imprecisas. En tal sentido, el entendimiento de las interrelaciones entre comunicación y gerencia pueden estar ofreciendo un campo de interés diferente al 
del los esquemas aislados y unidireccionales tradicionales. La información y el conocimiento están constituyendo el nuevo epicentro de las transformaciones en to que muchos autores han explicado como la era de la información (Castells, 1997; Wilson, 1998), o la era postindustrial o postcapitalista (Drucker, 1993).

Aclaremos brevisimamente la idea de posmodernidad. Es un concepto que atraviesa a diversas disciplinas (filosofía, sociologia, arquitectura, arte, educación, comunicación), y que nos habla de un estado de desarrollo de las cosas, de un cierto modo de reflexionar, de actuar y de ser; de una forma de pensamiento, de una visión del mundo, de una gran hipótesis de la vida, de un quiebre en la ruta de la modernidad. En sentido estricto, nos habla de algo nuevo, de un gran cambio de paradigmas, de nuevas oportunidades. Eso es tan cierto como que el término posmodernidad se puede volver, para algunos, imple. invertido, poli sémico o multidinisisiounal, mientras que para otros se puede convertir en una moda. Eso es parte de lo interesante del debate: la diversidad y la complejidad.

Tenemos tres conjuntos de datos claves para percibir esta nueva dimensión de posmodernidad en el mundo organizacional: 1) sentimos que las ideas y sus fronteras se hacen difusas, imprecisas e inciertas: es decir, comienzan a ganar terreno las incertidumbres y las paradojas, al tiempo que se acrecienta el debate entre lo que ha sido y lo que puede ser en el mundo de la organización; 2) sentimos alguna desconfianza y desilusión en torno a la ciencia tradicional y al futuro lineal y previsible: es decir, comienzan a moverse los paradigmas y a impo- nerse una reinterpretación del futuro organizacional, surgen nuevos enfoques para explicar la construcción y dirección de la organización, al tiempo que se acepta una crisis del discurso gerencial, $y, 3)$ sentimos que los caminos únicos y tradicionales pueden tener poco sentido ante el caos y la complejidad. es decir, comienzan a ganar terreno las visiones complejas y transversales, los nuevos enfoques extra disciplinarios al tiempo que se acrecienta el sentido de oportunidad. En definitiva, se nos mueve el piso de la seguridad ideológica y científica, mientras que las organizaciones comienzan a desplazarse de lo rígido a lo versátil, de lo físico a lo virtual e intangible, de lo previsible a lo imprevisible, de lo gobernable a lo ingobernable, de lo administrativo a lo informacional. Han cambiado las reglas del juego y es entonces cuando nos estremecen los vientos posmodernos para ayudar a explicar las nuevas transformaciones organizacionales.

Estas tres percepciones afectan a las organizaciones y a sus procesos gerenciales y comunicacionales. Muchas analogias y reducciones, como las mecánicas, pueden ser ahora insuficientes para interpretar diversos fenómenos en la organización y en su comunicación.

Aquel análisis científico altamente racionalista, mecanicista, que dio origen al pensamiento administrativo gerencial, ha entrado en crisis (Llano, 94), está colapsando, al menos desde la perspectiva de la posmodernidad: no todo puede ser explicado con las mismas rutinas y certezas, ya que éstas pueden ser insuficientes y pueden llegar a constituir una camisa de fuerza para la observación, interpretación y dirección de la vida en las or- 
ganizaciones (Knights, 1992). Las organizaciones y las empresas pueden estar dependiendo menos de las finanzas que de la información. Ello nos lleva a suponer que puede estar apareciendo un abismo entre los movimientos de transformación organizacional y el aparataje conceptual para comprender y explicar tales movimientos.

La importancia creciente de la información y de la comunicación, la virtualización de las organizaciones, la complejidad del cambio y de las nuevas ideas, y un crecimiento de las incertidumbres y las paradojas, así como de la acción direccionadora de la comunicación, parecen haber sorprendido y desnudado a la gerencia tradicional (Rojas et al, 1995). En la actual sociedad de las comunicaciones puede haber una crisis de dirección en aquella gerencia más industrial que postindustrial, más centrada en lo tangible que en lo intangible, más centrada en la aparente facilidad que en la aceptación de la complejidad y el caos, más centrada en la confiabilidad sobre el futuro que en la variabilidad de éste. Ha sido una gerencia lineal que hoy se debate en un mundo multidimensional en red. Pero la idea misma del futuro, fundamental para la gerencia, puede estar en crisis por el incremento en la imprevisibilidad, por el caos y por la complejidad.

Dentro del mundo organizacional normalmente las relaciones entre Comunicación, Gerencia y Futuro se plantean en funciones dependientes y no de modo integrativo que es la perspectiva teórica que deseamos asumir. Es decir, el sentido de una triada. El mundo es complejo y es un riesgo interpretar las relaciones bajo la unidireccionalidad y monodiscipli- nareiedad que ha prevalecido. Deseamos centrar la atención desde la perspectiva de la complejidad (Morín, 1990), intentando buscar las oportunidades y retos en las nuevas transformaciones que la sociedad ofrece a las organizaciones (Castells, 1997; Horgan, 1998). Pero apenas podemos percibir algunos signos de cambios. Mirando hacia el futuro la ciencia avanza rápidamente y también sus impactos y autotransformaciones (Stevenson \& Inayatullah 1998, Horgan 1998). Las fronteras disciplinarias entran en crisis, como las verdades y los métodos. Vivimos momentos de disolución, resolución y reconstrucción (Norgaard, 1994) de diversas instituciones y organizaciones, y una forma de abordar el entendimiento de estas transformaciones es mediante la interrelaciones entre las ideas de Comunicación, la Gerencia y el Futuro.

\section{La naturaleza de la tríada: comunicación, gerencia y futuro}

La idea de triada viene de triangularidad, de tres elementos interrelacionados. Ya Foucault (1980) nos habló de una importante tríada en el ámbito organizacional: "el poder, el conocimiento y las normas" como unas fuerzas entrelazadas al interior de la organización, como un trío inseparable. Nosotros centramos la atención en otra tríada en la que ahora se relaciona el proyecto de la organización: su futuro. La gerencia dirige hacia el futuro y se soporta en la comunicación como proceso; la comunicación dirige, como la gerencia, al integrar voluntades; la gerencia encamina acciones hacia el futuro, la co- 
municación integra las acciones sobre una visión del futuro. El futuro se presenta como el proyecto de la organización, de la gerencia y de la comunicación. Resulta difícil imaginar una acción gerencial sin idea de futuro. También resulta dificil pensar una comunicación sin direccionalidad o sin sentido de futuro. Gerencia y comunicación, nos dirigen al futuro, lo construyen, aunque sea en proyecto (Rojas y Arapé, 1998). Comunicación, Gerencia y Futuro se integran en conceptos y acciones. Conforman una tríada no fácil de captar pero real. Constituyen un todo sinérgico que la ciencia ha fragmentado para su entendimiento pero que ahora intentamos observar como un todo.

Los cambios que actualmente se generan desde el mundo comunicacional nos permiten denominar a la era actual como la era de las comunicaciones. El universo de la comunicación envuelve hoy a toda la sociedad ofreciendo nuevos horizontes, para el saber, el hacer y el ser. Ello incluye a las organizaciones. Apenas comenzamos a tomar conciencia de su valor transformador (Uchi$\mathrm{da}_{1}$ 1996). No podemos dejar de estar en la comunicación en el mundo de las comunicaciones. Comunicación y gerencia van de la mano en la vida organizacional $y$ empresarial hacia el futuro. Difícilmente podemos imaginar una acción directiva sin comunicación o una comunicación exenta de dirección. También resulta difícil imaginar la construcción de algún un futuro sin acción directiva y sin acción comunicativa.

La comunicación y la gerencia constituyen una compleja paradoja; se esgrimen como causa real o aparente de diversos problemas en la vida organiza- cional, y, a la vez, se presentan como la esperanza de las diversas soluciones. En esto, obviamente, hay una parte de mito y una parte de realidad. Dentro de las organizamos convivimos en torno a la comunicación. Gerenciamos en torno a la comunicación. Las organizaciones utilizan gerencialmente a la comunicación en sus dimensiones interpersonales, grupales, corporativas y sociales. La organización vive a partir de la comunicación. La comunicación se convierte en expresión de la organización. Pero también podemos pensar que la organización es un producto de su cultura comunicacional y en este sentido se convierte en una metáfora de su comunicación. Así como la empresa es el producto de su gerencia; la organización empresarial y su gerencia pueden ser concebidas como producto de la acción transformadora de la comunicación.

Pudiéramos precisar aún más y denominar a esta era como la era de las oportunidades y retos que nos plantea el mundo de las comunicaciones. La organización es, al menos en hipótesis, la realización y/o proyección de sus oportunidades comunicacionales. Las nuevas realidades indican que muchas empresas cifran sus riquezas en intangibles como la información, la comunicación y el conocimiento. Eso parece ser un signo característico de la posmodernidad. En esta era de las comunicaciones las organizaciones y empresas juegan con reglas diferentes a las empresas de la era industrial. Las claves empresariales no están ya en la estructuia mecánica de los procesos estandarizados. Las nuevas claves pueden estar en el reconocimiento de los valores intangibles de la información, el conocimiento y su comunicación, en el valor 
Comunicación, Gerencia y Futuro

Rojas, L.; Arapé, E.; Mujica, M.; Rodríguez, R.; Lara, C.; Durand, E.; Ugas, G.

de la comunicación para generar y agregar valor, en el desarrollo de ventajas estratégicas con base a la información y la comunicación. En la fuerza transformadora de la información y la comunicación dentro de la vida de las organizaciones.

La practica comunicacional es altamente compleja e incluye desde lo humano hasta lo social, desde lo físico hasta lo virtual. La vida social y organizacional es consecuencia de las acciones comunicacionales (Habermas, 1997, 1998). Si la comunicación fuera más fácil y sencilla tendriamos otras organizaciones más cordiales, y sensatas; tendriamos otra sociedad más habitable. Pero también la comunicación total es una fantasía, una ilusión. Dentro de las organizaciones quedan zonas incomunicadas y descomunicadas. No podemos simplificar la magnitud del problema comunicacional, tampoco mitificarlo: ambos extremos podrían ser producto de los propios errores de la ciencia (Horgan, 1998). Tenemos la realidad que comunicacionalmente hemos construido y el proyecto de futuro para la organización puede depender de la comunicación. Sin embargo, parece que estamos en deuda con la comprensión y el entendimiento de la comunicación, $y$, obviamente, debemos recordar que la comunicación, aun en la empresa, tiene como centro al hombre, a la persona; y que tal vez estemos en deuda ya no sólo con el entendimiento de la comunicación en general, sino con la cultura comunicacional.

Desde esta perspectiva la gerencia que no atienda suficientemente a la comunicación (Llano, 1994). Dentro de las organizaciones se puede estar experimentando una brecha entre el entendi- miento del desarrollo de las tecnologías de comunicación y el entendimiento de la comunicación humana y social. Puede haber también una enorme brecha entre la gerencia y la comunicación dentro de la organización tradicional. Puede haber una creciente brecha entre, por una parte, la empresa moderna y su gerencia moderna, y por la otra, la empresa postmoderna y su gerencia también postmoderna, aun cuando esta últimas sean para muchos una hipótesis. Creemos que el abordaje, el tratamiento y la interpretación de la tríada Comunicación, Gerencia y Futuro, puede ayudarnos a entender cómo saldar estas brechas.

Resumiendo, tenemos que desde el interior de la triada hay varias líneas de interés que llaman nuestra atención y que van desde lo simple a lo complejo: 1) la utilidad práctica que hará la gerencia respecto a la comunicación en su camino al futuro, 2) el creciente papel direccionador de la comunicación, 3) la construcción de futuros desde la comunicación y/o desde la gerencia, 4) la interpretación gerencial de la empresa vs. la interpretación comunicacional de la misma, 5) la construcción y disolución de la organización mediante la gerencia y/o la comunicación, 6) los grandes problemas que el futuro nos anuncia para interpretar la nueva comunicación o la nueva gerencia, $y$, entre otros, 7) el déficit intelectual que padecemos para percatarnos de los cambios, para aprovechar las oportunidades y para interpretar las nuevas realidades. Estas lineas pueden conducirnos por diversos caminos en un gran mapa que ahora necesitamos sintetizar.

Atravesando estas grandes líneas de interés, confrontamos la perspectiva 
teórica con la perspectiva práctica de este trabajo, si es que en realidad se pudiera establecer tal frontera. La perspectiva teórica está representada, por una parte, con aquellos tres conjuntos de datos clave que nos ayudan a percibir la cuestión posmoderna en la organización (véase primeras páginas), y, por la otra, con las respuestas a las preguntas que orientaron la revisión de literatura. La perspectiva práctica estaria representada por una parte con las interpretaciones al conjunto de respuestas a las tres preguntas centrales que orientaron las entrevistas (véase metodología) y por la otra con las matizaciones y debates en los Seminarios referidos. Tras la confrontación y sintesis logramos identificar cinco grandes problemas o ejes a través de los cuales podemos plantear visiones interpretativas de la tríada Comunicación, Gerencia y Futuro.

\section{Primer problema: El cambio incremental}

El primer gran problema que nos encontramos al relacionar Comunicación, Gerencia y Futuro en el mundo organizacional, es el fenómeno del cambio incremental. Es decir, el cambio tecnológico, el cambio de ideas, de realidades y de agendas de problemas, parece tan rápido, que se acelera el cambio en el valor y uso de la información y de la fuerza transformacional de la comunicación. La vida organizacional está cambiando aceleradamente por el impacto de los cambios producidos en el mundo de la información y de la comunicación (Castelis, 1997). La lectura del cambio comunicacional en el mundo de hoy, expresada al menos en sus oportunidades y retos, la responsabilidad transformadora de la comunicación, y la esperanza que tenemos en ella para el futuro, en organización y en la sociedad, poco se parecen a la lectura del mundo comunicacional de una década atrás. Parece que el mundo comunicacional y organizacional está cambiado tanto y tan rápidamente que nos cuesta entender lo que está sucediendo. El camino hacia la informatización de la sociedad, hacia la globalización, hacia las particularizaciones, hacia la obsolescencia y hacia la diversidad, es tan rápido que parece difícil mantenerse al día en su captación. El mundo ha cambiado vertiginosamente con una comunicación que ha ofrecido una compresión y una expansión recomponiéndolo todo. El mundo, paradójicamente, parece más pequeño y más grande al mismo tiempo. Lo que antes era lejano ahora es cercano y viceversa. Se afecta aceleradamente al tener, al hacer, al saber y al ser. Nunca antes las oportunidades comunicacionales habian sido tan grandes. Nunca antes la comunicación nos habia traído tantas paradojas.

El crecimiento acelerado de Internet y los Webs modifican la realidad a cada instante. Tiempo y espacio se comprimen y se expanden. La organización se mueve de lo real a lo virtual, igual sucede con la gerencia. Se mueven siguiendo el camino de los intangibles como la información, el conocimiento y su comunicación. Esta primera gran tendencia implica, al menos, tres formas de participación para los trabajadores, los gerentes y las empresas en general: 1) los que están, aunque sea por un instante, en la vanguardia de los cambios comunicacionales, desde lo tecnológico hasta lo cultural; 
Rojas, L.; Arapé, E.; Mujica, M.; Rodríguez, R.; Lara, C.; Durand, E.; Ugas, G.

es decir en la creación o promoción del cambio, 2) los que intentan sobrevivir y se mantienen a un ritmo elemental, no dominan la última tecnología o no tienen una amplitud cultural para entender los cambios comunicacionales, están en lucha permanente para sobrevivir; y 3) los que se han quedado fuera de la velocidad de los cambios o no han podido mantenerse, digamos, competitivos en el mundo de las oportunidades comunicacionales. Estos últimos podrían ser los espectadores de los cambios.

Los cambios son tan acelerados que las organizaciones actuales se están pareciendo poco a las organizaciones tradicionales: horizontalidad en vez de verticalidad, outsourcing en vez de concentración, globalización y adaptaciones de localización en vez de estandarizaciones, virtualización en vez de organizaciones físicas, organización en red vs. Departamentalización, en fin, desplazamiento hacia formas posmodernas. Las organizaciones están luchando paradójicamente entre las fuerzas de estabilidad tradicional para mantener el poder y el control, y las fuerzas transformadoras para cambiar aceleradamente, nos dicen Howard y Geist, (1995) siguiendo a los estudios de Giddens. Muchas organizaciones educativas tradicionales están dando paso a las organizaciones virtuales (Silvio, 1998). El mundo de los negocios comienza a ganar terreno en las nuevas formas de organización altamente cambiantes, versátiles y virtuales.

Los cambios afectan al concepto tradicional de trabajo desplazándonos por ejemplo hacia el teletrabajo. La idea de puesto de trabajo ha sido superada. Los procesos son diversos $y$ las esperanzas están cifradas más en la oportunidad de un trabajo que en éste mismo. Pero una preocupación se desprende de esta tendencia: el cambio para las organizaciones y la gerencia puede ser tan acelerado que predecir el futuro sería una aventura. La linealidad hacia el futuro, su previsibilidad no son tan exactos en esta sociedad postindustrial y posmoderna. $Y$ esto sacude las bases de la gerencia tradicional.

El cambio incremental significa obsolescencia creciente y acelerada tanto en lo real y lo tecnológico, como en lo intangible, los conceptos y la información. Las verdades sólo lo son mientras se escriben. Los esquemas interpretativos también varian constantemente. Las organizaciones cambian a cada hora como las bolsas de valores. El tiempo se comprime y los ciclos de los procesos son cada vez más cortos. El tiempo, la velocidad y consecuentemente el espacio se perciben de un modo diferente desde la perspectiva del cambio incremental. Ello conduce a la necesidad de incrementar la rapidez de adaptabilidad, la velocidad de Innovación (Jackson el al, 1995) y de creación de una cultura de la creatividad (Murakami, et al, 1993).

El cambio incremental, y su fuerza transtormadora, puede originarse desde la acción gerencial o desde la acción comunicacional. Pero escapan de ellas y se superponen. Comunicación y Gerencia generan el cambio hacia el futuro y son objeto del cambio acelerado. $O$ cambian o perecen.

\section{Segundo problema: El gobierno de la infocomunicación}

El segundo gran problema relaciona la importancia creciente y el papel pre- 
ponderante de la información y la comunicación. Nadie discute que el mundo gira hoy en torno a la información y la comunicación: La sinergia información- comunicación parece gobernarlo todo. Lo que sucede diariamente en la banca internacional, en la TV, o en Internet, es prueba de ello. El mundo comunicacional se ha globalizado. Nunca antes habíamos dependido tanto en niveles personales $y$ empresariales de la información y la comunicación (Páez, 1992). Información y comunicación constituyen una sola entidad: infocomunicación. La frontera entre ambas es relativa, como lo son las fronteras disciplinarias. La infocomunicación constituyen una especie de metagobierno invisible, o virtual, sobre todo desde la perspectiva de la sociedad red (Castells, 1997). Se gerencia con base a la información y a la comunicación. La organízación es gobernada por su saber informacional y comunicaciónal en tanto la organización es metáfora de la cultura infocomunicacional. El conocimiento y el poder devienen de la infocomunicación, también la inteligencia empresarial y su acción transformadora. El Profesor Figueroa (1995) nos comenta desde México: "No existe proceso de desarrollo sin una visión del mundo y hoy la principal visión del mundo que recibimos la transmiten los medios electrónicos".

Muchos procesos de trabajo, o la actuación misma del trabajador, sea médico, educador o químico, dependen como nunca antes del papel preponderante de la información y la comunicación. Muchos de ellos se están reconvirtiendo hacia una dimensión informaciona: y comunicacional. Las capacidades para transformar partiendo de la información y la comunicación son cada vez más importantes. La producción de información y conocimiento, y su comunicación, cada vez más instantánea y múltiple, se convierten en un asunto clave para la construcción de la sociedad del siglo XXI (Aronowitz el at, 1998). La Información y la comunicación, en su sentido general, son las nuevas fuentes de producción, poder y riqueza. Son también, la clave para reinterpretar a la organización desde una perspectiva posmoderna.

Estar gobernados por la Infocomunicación significa que ésta dirige diversas actividades de la vida: negocios, educación, entretenimiento, trabajo o evasión en un complejo mix que antes no existía. Ha borrado fronteras, ha creado nuevas realidades, con nuevos peligros y oportunidades. Inclusive afecta a la geopolítica de los países, a los Estados, a los partidos políticos, y a las empresas. Infocomunicación significa la integración en redes de comunicación en busca de información para comunicarla y transformar a partir de este eje. Pero es distinto de lo tecnológico. Está apoyado en la tecnología, pero sobre todo en un nuevo saber $y$ un nuevo ser. La información y la comunicación han dejado de ser un recurso más como lo era en la empresa moderna para convertirse en el epicentro de las transformaciones en las nuevas organizaciones posmodernas.

Ahora la clave está en el acceso diverso, libre y voluntario a la información, en su mariejo, en la capacidad de relación y de comunicación. Podriamos decir en Li id nueva inteligencia informacional y comunicacional, independientemente de su relación con las clases sociales o con las clases informacionales. Es la cultura de la 
Rojas, L.; Arapé, E.; Mujica, M.; Rodriguez, R.; Lara, C.; Durand, E.; Ugas, G.

información y de la comunicación extra aula, y el aprovechamiento de esas oportunidades que nos brinda la red, 10 que puede marcar la diferencia entre los niveles culturales de los estudiantes y los docentes dentro de las nuevas organizaciones basadas en el conocimiento. $O$ dicho con mayor precisión: la diferencia estriba hoy en estar o no integrado al mundo infocomunicacional. Lo mismo sucede en la organización: la empresa comienza crecervertiginosamente en sus bordes, en la virtualidad. Gran parte del trabajo to hacemos desde las casas o a través de la red, en forma de teletrabajo o sencillamente fuera de la organización. Comienza a desaparecer la cultura del puesto de trabajo y de la gerencia controladora de horario. Pero aquí hay un déficit. Expertos en comunicación y gerencia parecen ir por caminos sindicales separados, parecen estar más preocupados por cuestiones puntuales que de estrategias globales.

La clave es que en la era de las comunicaciones debemos actuar con el instrumental técnico y conceptual propio de estos tiempos. Aquí el reto que vemos está asociado a la comprensión intelectual para entender el papel preponderante de la información y la comunicación en el mundo actual y consecuentemente al saber actuar en un mundo más regido por intangibles o virtualidades que por dominios materiales.

\section{Tercer problema: La crisis de los paradigmas}

El tercer gran problema es lo que denominamos la crisis de los paradigmas, la crisis de las fronteras entre los modelos. La tendencia nos indica que ha- cia el futuro las fronteras conceptuales parecen superponerse y los modelos tradicionales de comunicación, empresa, educación y diversión parecen ser insuficientes para explicar las nuevas realidades. Alli tenemos por una parte: 1) fenómenos nuevos como la expansión e intensificación de la comunicación, ejemplificadas con la globalización comunicacional o la potenciación de comunicaciones locales y su incidencia en los procesos sociales, 2) el fenómeno de la aparición de nuevos medios como los multimedias y las Webs, el fenómeno de la sociedad en red, o el fenómeno de virtualización, 3) las nuevas relaciones hombre-tecnología, la adicción a la navegación, o el teletrabajo. $Y$ entre otros ejemplos de la crisis de los modelos tenemos el caso de la TV e Internet y su ámbito cultural y educativo: saltamos de lo local a mundial al instante, saltamos de integración alienante a una integración liberadora. Hoy la alienación puede no estar en los medios, viejos o nuevos, como se afirmaba en el paradigma de los años 60 , es posible que esté fuera de ellos, los medios son los medios de integración de la nueva sociedad comunicacional. No estar integrados puede significar sencillamente no estar en el mundo. Este es el cambio de paradigma. Lo que antes parecía oponerse a la educación hoy parecen guiarla de alguna manera: los medios, sobre todo los nuevos medios. Lo que antes parecía oponerse a la empresa, hoy parece guiarla: el trabajo desde fuera.

Definitivamente las nuevas realidades parecen poner en jaque a los modelos que hasta ahora habiamos venido utilizando para abordar el complejo mundo de la comunicación y la gerencia en las 
organizaciones. El saber y razón académica puede está fuera de la organización empresarial y educativa. Están en el mundo infocomunicacional intangible. Paradójicamente en todas parte y en ninguna, están en el mundo virtual que no es real para la ciencia positiva tradicional, ni se puede gobernar con la gerencia tradicional.

La tercera ruta de problemas nos indica la crisis de los modelos organizacionales. La institución educativa tradicional, por ejemplo, con sus estilos educativos está en crisis no sólo financiera, sino principalmente de sentido (Duch, 1997). Lo mismo sucede con organización empresarial: se pueden estar logrando los objetivos pero es indiscutible una crisis de sentido. La razón filosófica de la empresa está divorciada de la razón pragmática. Las insatisfacciones humanas y sociales, las ilusiones y desilusiones, el desempleo, la inseguridad, el pobre bienestar, y la disolución del medio ambiente, no son temas que escapan a la responsabilidad empresarial. La gerencia puede estar en deuda con la empresa como éstas están con el hombre y la sociedad. ¿Acaso el proyecto histórico de la escuela y la empresa moderna han logrado el bienestar ofrecido? ¿Acaso no se estarán disolviendo o metamorfoseando hacia una organización postmoderna en busca de un nuevo sentido? En muchas organizaciones parece que se ha perdido el rumbo y se siente un enorme malestar que se evidencia en frustraciones personales e insatisfacciones sociales. Está en crisis el modelo de enseñanza aprendizaje basado en el saber del docente ilustrado. Está en crisis el modelo gerencial centrado en el éxito, el poder o la autori- dad del gerente. El paradigma clásico del trabajador supervisado, gerenciado está en crisis. Un trabajador conectado independientemente a la sociedad en red puede tener más acceso a la información, al saber y a las capacidades creativas y transformadoras que un gerente tradicional no conectado. Muchos gerentes pueden perder el tiempo y las oportunidades tratando de mantenerse en el poder siguiendo el camino tradicional mientras otros avanzan hacia el poder mediante el poder de la infocomunicación. $Y$ esto es un cambio de paradigma. Mucha gente invierte como nunca antes en educación formal, en formación continua, en perfeccionamiento profesional o directivo, en capacitación, o en navegación, sencillamente para tener más información y nuevas relaciones comunicacionales que le permitan adecuarse a los cambios de paradigmas.

Pero hay otros cambios internos en el paradigma de la adquisición del saber transformador en la organización. La adquisición está signada por el cambio incremental, por la responsabilidad individual y el trabajo en equipos, y por posibilidades y capacidades para acceder al mundo infocomunicacional. Tal vez hoy la gerencia comienza a desplazarse de una función centrada en la planificación y supervisión a otra centrada en la ideación de futuros y en la facilitación de aprendizajes. Tal vez hoy en estas realidades de la sociedad comunicacional el mejor gerente no sea el que mejor mande, como que el mejor docente no sea el que mejor explique. Tal vez el mejor gerente sea como el mejor educador, el que mejor sepa dirigir al trabajador ( $y$ alumno) hacia la información y el conocimiento, el que 
mejor sepa asociarse a la búsqueda e interpretación de la información, el que mejor sepa integrarse en equipo a la sociedad de las comunicaciones y generar desde alli las transformaciones. Una cuestión clave es entender que las organizaciones son recolectoras y productoras de información y conocimientos, son comunidades de aprendizaje, son comunidades comunicacionales.

Crisis de los modelos significa que muchas formas de enseñar, aprender y trabajar pueden estar fracasadas por insuficientes, inadecuadas $\circ$ atrasadas. Significa que las nuevas formas de trabajo pueden ser paradójicamente, globales y locales en cuanto a tecnología y en cuanto a cultura, y pueden ser tal vez más versátiles. Crisis de paradigmas significa que los modelos de organizacionales y gerenciales están en crisis, y que está en crisis lo que estamos aprendiendo para construir y dirigir las futuras organizaciones. Y por supuesto, que está en crisis la idea de futuro.

Pero también crisis de paradigmas puede implicar la disolución de los elementos de la triada si se observan aisladamente, precisamente por la desilusión que cada uno de ellos nos presenta. Después de tal disolución sólo podríamos abordar y recuperar los elementos recomponiéndolos en una complejidad.

\section{Cuarto problema: El juego de las paradojas}

El cuarto gran problema con que se encuentra la organización y la gerencia hacia el futuro es lo que podríamos definir como el juego de las paradojas. Esto es: cada día, en este cambio acelerado de todo, donde la información y la comunicación parecen gobernarlo todo, donde crece la complejidad en comunicación, crece consecuentemente el juego de las paradojas. La dirección del cambio puede ser doble, aparentemente contradictoria, compleja o confusa, y esto implica una nueva forma de organización y de actuación gerencial, diferente a cuando las cosas parecian previsibles, claras, rutinarias o sencillamente dirigibles. Veamos algunas paradojas: 1) la misma tendencia que nos empuja hacia globalización nos permite potenciar la vida municipal, 2) mientras más comunicados estamos a través de la tecnología y la globalización más crece la incomunicación y descomunicación en lo humano, 3) mientras más esperanzas tenemos en las contribuciones comunicacionales menos parece contribuir ésta a aumentar la calidad de vida o el bienestar social, 4) mientras crece la responsabilidad de la comunicación, la gerencia o la educación como causante de los hechos que no nos gustan en esta vida, aumenta la esperanza en que sólo a través de ellas podamos resolver las cosas que han salido mal, 5) mientras más gerentes se forman mayor es la crisis de los paradigmas empresariales y gerenciales, o6) mientras más crece la educación más ignorancia tenemos (Ugas, 1997). La cuestión de las paradojas puede estar relacionadas con la frontera de nuestros conocimientos donde los límites entre el razonamiento y la especulación pueden estar signados por la incertidumbre (Prigogine, 1997).

En el mundo organizacional la comunicación y la gerencia, desde la humana hasta la globalizada, se tornan más complejas y paradójicas, parecen ser 
más culpables y a la vez más esperanzadoras, parece fragmentar al grupo al tiempo que reorganiza a la colectividad, parece que desencuentran al hombre pero reencuentran al individuo. Ese es el crecimiento del juego de las paradojas en torno a la sociedad comunicacional, en torno al mundo organizacional. Comunicación y gerencia están llenas de certidumbres e incertidumbres, de ambivalencias, de mitos y realidades, de ser y no ser, no como dilemas sino como paradojas. Son y a la vez no son. Las cosas no son tan fáciles como parecen y la verdad puede estar, momentáneamente, en la interpretación de las palabras. "El mundo será un lugar lleno de confusión en las próximas décadas" sentencia Charles Handey hacia el final de su obra La Edad de la Paradoja. Ello es cierto tanto para la comunicación como para la gerencia. Ello puede hacer más complicado el entendimiento de la actual era de las comunicaciones que ya es dificil comparar con modelos reduccionistas. La paradoja es la incertidumbre entre valores que pueden ser ciertos en una situación caracterizada por la complejidad y la relatividad de la interpretación. Nuevamente el valor de la información para predecir un futuro cada vez más impredecible.

Desde las paradojas tenemos un conjunto de oportunidades relacionadas con la amplitud para buscar soluciones e interpretaciones a las nuevas realidades. Las nuevas búsquedas pueden ser más libres, multidireccionales y de una certidumbre tan relativa como nos enfoquemos dentro de la paradoja. La línea de retos puede estar relacionada con el aprendizaje de una forma de pensar y actuar más cercana de la incertidumbre que de la certidumbre, con el desarrollo de un pensamiento complejo para actuar ante las paradojas. Tal vez en esto la superación de las fronteras disciplinarias y la movilización hacia un pensamiento transdisciplinario pueda darnos más luz ante problemas como los de la tríada Comunicación, Gerencia y Futuro.

Para la organización, su cultura, y en particular su gerencia el asunto es más dramático. El camino hacia el futuro ha dejado de ser conocido y predecible para ser incierto y paradójico. ¿Acaso las organizaciones, empresariales por ejemplo, y la gerencia están entendiendo que se mueven hacia una inteligencia más caótica e intuitiva?

\section{Quinto problema: El déficit intelectual}

El quinto gran problema es el que denominamos el crecimiento del déficit intelectual. No por tener mayor acceso a más información, o por tenerla, aumenta nuestra comprensión de los cambios y de las nuevas realidades. Las nuevas realidades comunicacionales y organizacionales son tan arrolladoras, tan complejas y tan rápidas, que parece que se va creando un desfase entre ellas y su comprensión. Por ejemplo: puede ser insuficiente explicar el avance de las comunicaciones sólo desde la perspectiva del avance de la tecnologia, como puede ser insuficiente creer que el saber y la razón para dirigir a la organización están sólo en la gerencia. El mismo fenómeno de i2K (el fenómeno informático del año 2000) es un ejemplo de este déficit, igual 
que los retos de la bioética, del cambio en los estilos de trabajo o de las transformaciones organizacionales. Otra evidencia del déficit intelectual puede ser el intento de explicar las nuevas realidades con viejos esquemas. El cyberespacio, los hipervínculos, la adicción a la navegación, no pueden ser entendidos con los esquemas de los años 60 . Inclusive su estudio y diseño metodológico tanto desde el punto de vista comunicacional como educativo comprende una gran deuda a la ciencia (Rojas y Arapé, 1996; Montiel, 1998). Las nuevas realidades comunicacionales $y$ organizacionales están requiriendo ser interpretadas y estudiadas desde al menos una perspectiva interdisciplinaria, 0 mejor transdisciplinaria, pero parece que en nuestro contexto continúan aferrados a perspectivas intradisciplinarias. Realidades complejas requieren análisis integrales complejos y generan pensamientos complejos, modelos complejos.

El déficit intelectual es el bloqueo para entender lo que está sucediendo, comprender el cambio incremental, el juego de las paradojas, el cambio de paradigmas y el papel de la infocomunicación. Podemos analizar la tríada desde la misma línea de la tradicional de la modernidad, como podemos estudiar temas de la posmodernidad con un aparataje moderno o posmoderno. Digamos que ese déficit intelectual es menor, en lo personal y en lo organizacional, si estamos cerca de la cresta de la ola, pero es dramáticamente mayor si el grueso de los actores-trabajadores se encuentran en al cola de los espectadores. En la medida en que el cambio que provoca el cambio incremental es más rápido, mayor es el riesgo de incrementar el déficit intelectual de las personas y la organización.

Aclaremos algo sobre el déficit intelectual. El hecho de que utilicemos nuevas tecnologías no significa que no tengamos el déficit intelectual para entender el nuevo mundo comunicacional y organizacional, o que sea suficiente para aprovechar plenamente las oportunidades de la sociedad comunicacional. El déficit intelectual es más cultural que técnico. El déficit lo podremos nivelar en la medida que desarrollemos nuestra cultura y comprensión del cambio estratégico que supone nuestra actuación transformadora en esta sociedad de la comunicación. Lo podremos nivelar en la medida en que actualicemos nuestro marco teórico y conceptual sobre lo que está sucediendo: no podemos intentar explicar el proyecto de la organización posmoderna y una gerencia posmoderna en una sociedad que tiende hacia lo posmoderno, con el instrumental teórico y conceptual de los esquemas modernos o modernos.

El déficit puede ser reconocido o ignorado, pero lo cierto es que cada día el mundo comunicacional y organizacional, y su percepción, se hace más complejo, más difícil de captar e interpretar. Posiblemente una vía para nivelar el déficit sea la superación de limites conceptuales y la integración de nuevos campos y nuevos enfoques para el saber

\section{A manera de conclusión}

Comunicación, Gerencia y Futuro constituyen una tríada que nos afecta a todos en el mundo organizacional y social, y una realidad de la que no podemos 
escapar en esta sociedad de las comunicaciones. Ante esto tenemos grandes problemas para reinterpretar las relaciones Comunicación, Gerencia y Futuro. Tal vez la mayor de las oportunidades sea la de aceptar el cambio del cambio. El peligro puede estar en negarnos a entender las nuevas realidades y las nuevas oportunidades. En nuestras manos está decidir hasta cuando retrasar nuestro ingreso personal a este nuevo mundo, o hasta cuando seguir viendo los problemas como los hablamos visto. Luego de entrar, tarde o temprano, a saldar el déficit por un instante y situarnos por otro instante en la cresta de la ola, tendremos el mayor de los retos: mantenernos en al juego del cambio acelerado intentaitu.. solventar nuevamente el déficit intelectual. Pero cuando ya no podamos con el juego del cambio y la actualización permanente en este mundo de paradojas y de globalización comunicacional seremos expulsados del juego y devueltos a la sociedad agraria o al mundo de la ecología doméstica para ver como espectadores la película del mundo de la era comunicacional sin que podamos entenderla a cabalidad.

Finalmente, el fenómeno de la tríada Comunicación, Gerencia y Futuro, es sólo una de las tantas sorpresas con las que comenzamos a toparnos en esta sociedad de las comunicaciones, y su complejidad una evidencia de las percepciones posmodernas. Su atención por parte de científicos y académicos en este pais es una muestra de cómo estamos afrontando en cambio y como intentamos nivelar el déficit conceptual. Tal vez, algo importante de la agenda pendiente sea un llamado de atención para abordar desde las universidades el estudio del impacto de las comunicaciones en la nueva organización y su gerencia, desde una perspectiva más amplia, más activa y más comprometida con la construcción del futuro. Tal vez una importante pista sea desprender la visión parcial e instrumental entre los elementos de la tríada y suplantarla por interpretaciones globales, integrales y complejas. Pero nos queda pendiente un último complejo de interrogantes. Sus respuestas negativas es sólo un reflejo hacia el pasado, sus respueszas positivas es una aventura; su sentido podría estar en un juego de las paradojas y la complejidad: ¿Acaso estaremos llegando al fin o disolución de la comunicacion, de la gerencia, de la organización, y del futuro? ¿Acaso no será una evidencia del déficit intelectual la simplificación conceptual en un mundo cada vez más complejo? ¿Acaso será necesario una lectura e interpretación posmoderna de la comunicación, la organización y la gerencia? ¿Acaso estaremos también en deuda con esta tríada? ¿O acaso estará ella en deuda con el hombre y la sociedad?

\section{Bibliografía citada}

Aronowitz, Stanley et al (1998) Tecnociencia y Cybercultura, Paidós.

Castells, Manuel (1997) La era de la informaclón, (volúmen 1: La sociedad red); Alianza, Barcelona.

Baudrillard, Jean (1968), El Sistema de los Objetos Siglo XXI México.

Derrida, Jacques (1972) Positions, Minuit Edit., Paris.

De Sousa Santos, Boaventura (1996) Introducción a una clencia posmoderna, CIPOST,UCV, Caracas. 
Rojas, L.; Arapé, E.; Mujica, M.; Rodriguez, R.; Lara, C.; Durand, E.; Ugas, G.

Drucker, Peter (1993) La Sociedad Postcapitalista, Norma, Colombia.

Duch, Lluís (1997) La educación y Crisis de la modernidad, Paidós.

Figueroa, José (1995) "Politicas de Comunicación ¿en una época de cambios?' En Comunicación, Globalización y Política (99-114), Universidad Autónoma del Estado de México, Toluca.

Follari, Roberto y Rigoberto Lanz (Comp) (1998) Enfoques sobre la posmodernidad en América Latina, Edit. Sentido Col, Pensamiento Transdisciplinario.

Foucault, Michel (1994) Hemenéutica del Sujeto, Edit. La Piqueta, Madrid,

Foucault, Michel (1980) Power / Knowledge, Gordon-Pantheon Edit, N.Y.

Gadamer, HansGeorge (1997) Mito y Razón, Paidós Estudios, Barcelona.

Green, Madeleine (1997) "Leadership and Ins" titutional change: a comparative view" Higher Education Management Journal, 9 (2) 135-146.

Habermas, Jürgen (1981) Modernidad versus postmodernidad, Picó Edit. Barcelona.

Habermas, Jürgen (1997) Teoria de la Acción Comunicativa, Cátedra, Barcelona.

Habermas, Jürgen (1998) Conciencia moral y acción comunicativa, Peninsula, Barcelona.

Handy, Charles (1996) La Edad de la Paradoja: dar sentido al futuro, Edic Apos. trofe, Barcelona.

Horgan, Jonh (1998) El Fin de la Ciencia, Paidós, Barcelona.

Howard, Liza y Patricia Geist (1995) "ideological positioning in organizational change: the dialectical of control in a merging organization" Communication Monographs Rev. 62 (2) 110-131.

Jackson, W. S. Caompbell, P. Write, y M. Kroll (1995) "The relationship of Incremen- tal innovations and performance: an empirical investigation of smalls business types" Journals of Business \& Entrepreneurship 7 (1) 1-14.

Knights, David (1992) "Changing spaces: the disnuptive impact off a new epistemological location for the study of management' Academy of Management Review, 17 (3) 514-536.

Llano, Carlos (1994) EI Postmodeernismoen al empresa, McGraw Hill, México.

Lyotard, Jean-Françoise (1979) La Condición Postmnoderna, Edic. Cátedra, Barcelona.

Merton, Hobert, M. Fisket y P. Kendall (1990 The focused interview, A manual of problems and procedures, Free Pres, New York.

Millar, A., V. Crute y O. Hargie (1992) Professional Interviewing, Routledge, Londres.

Montiel, Maryalejandra (1998) "Innovación Tecnológica, Globalización de la Información y Nuevos Medios de Comunicación Social", Tesis de Maestria, Inédita, U. del Zulia.

Morín, Edgar (1990) Introducción al pensamiento complejo, Gedisa, Barcelona.

Murakami, T., et al (1993) Estrategia de la creación, Edit. Panoráma, México.

Norgaard, Asbjord (1994) "institutions and Post-modernity in IR: the "new" EC" Cooperation and Conflict Journal 29 (3) 245-287.

Páez, Iracet (1992) Gestión de la Inteligencia, Inst. de Estudios del Conocimiento, USB-Monte Avila Edit. Caracas.

Prigogini, llya (1997) El fin de las certidumbres, Taurus, Barcelona.

Rojas, Luis Rodolfo; J. Mundet, y E Arapé (1995) "Empresa y Comunicación: Hacia una teoria del postmanagement"Revista de Ciencias Sociales, Fac. Economia LUZ. 
Rojas, Luis Rodolfo y Elizabeth Arapé (1996) "Hacia la postcomunicación" Revista Quark, № 4, Universidad Pompeu Fabra, 64-80.

Rojas, Luis Rodolfo (1998) "La formación en comunicación en algunas maestrias en gerencia de empresa", Revista Venezolana de Gerencia, $3(5) 73$ 91.

Schatzman, L. y A. Strauss (1973) Field Research. Strategies for a natural sociology. Englewood- Prentecy Hall, New York.

Silvio, José (1998) "La virtualización de las universidades" Ponencia en Visionarios 98, Caracas, 1998. CD Visionarios 98.
Stevenson, Tony \& Sohail Inayatullah (1998) "Futures-oriented writing and research" Future (journal) 30 (1) 1-2.

Uchida, Donna et al (1996) Preparing Student for the $21^{\text {st }}$ Century, American Association of Scholl Administration, Virginia.

Ugas, Gabriel (1997) La Ignorancia Educada, Est. Epistemológicos, ULA-Táchira

Valles, Miguel (1997).Técnicas Cualitativas de Investigación Social, Edit. Sintesis, Madrid.

Wilson, Ernest (1998) "Inventing the Global Information Future ${ }^{n}$ Futures (Journals) 30 (1) 23-42. 